\title{
Translational informatics approach for identifying the functional molecular communicators linking coronary artery disease, infection and inflammation
}

\author{
ANKIT SHARMA $^{1-3}$, MADANKUMAR GHATGE ${ }^{1,3}$, LAKSHMI MUNDKUR $^{4}$ and RAJANI KANTH VANGALA ${ }^{1,2}$ \\ ${ }^{1}$ Proteomics and Coagulation Unit; ${ }^{2}$ Emmanuel Key Bioinformatics and Biostatistics Unit, Thrombosis Research Institute, \\ Bangalore, Karnataka 560099; ${ }^{3}$ Manipal University, Manipal, Karnataka 576104; ${ }^{4}$ Molecular Immunology Unit, \\ Thrombosis Research Institute, Bangalore, Karnataka 560099, India
}

Received August 27, 2015; Accepted February 18, 2016

DOI: $10.3892 / \mathrm{mmr} .2016 .5013$

\begin{abstract}
Translational informatics approaches are required for the integration of diverse and accumulating data to enable the administration of effective translational medicine specifically in complex diseases such as coronary artery disease (CAD). In the current study, a novel approach for elucidating the association between infection, inflammation and CAD was used. Genes for CAD were collected from the CAD-gene database and those for infection and inflammation were collected from the UniProt database. The cytomegalovirus (CMV)-induced genes were identified from the literature and the CAD-associated clinical phenotypes were obtained from the Unified Medical Language System. A total of 55 gene ontologies (GO) termed functional communicator ontologies were identified in the gene sets linking clinical phenotypes in the diseasome network. The network topology analysis suggested that important functions including viral entry, cell adhesion, apoptosis, inflammatory and immune responses networked with clinical phenotypes. Microarray data was extracted from the Gene Expression Omnibus (dataset: GSE48060) for highly networked disease myocardial infarction. Further analysis of differentially expressed genes and their GO terms suggested that CMV infection may trigger a xenobiotic response, oxidative stress, inflammation and immune modulation. Notably, the current study identified $\gamma$-glutamyl transferase (GGT)-5 as a potential biomarker with an odds ratio of 1.947 ,
\end{abstract}

Correspondence to: Dr Rajani Kanth Vangala, Proteomics and Coagulation Unit, Thrombosis Research Institute, 258/A Bommasandra, Hosur Road, Bangalore, Karnataka 560099, India

E-mail: rajani@triindia.org.in

Abbreviations: CAD, coronary artery disease; MI, myocardial infarction; CMV, cytomegalovirus; GO, gene ontology; FCOs, functional communicator ontologies; GGT-5, $\gamma$-glutamyl transferase 5; ASP, average short path; ND, node degree

Key words: translational informatics, coronary artery disease, infection, $\gamma$-glutamyl transferase, risk prediction which increased to 2.561 following the addition of CMV and CMV-neutralizing antibody (CMV-NA) titers. The C-statistics increased from 0.530 for conventional risk factors (CRFs) to 0.711 for GGT in combination with the above mentioned infections and CRFs. Therefore, the translational informatics approach used in the current study identified a potential molecular mechanism for CMV infection in CAD, and a potential biomarker for risk prediction.

\section{Introduction}

Coronary artery disease (CAD) is a major cause of morbidity and mortality worldwide, with particularly high levels in India. The predisposing risk factors identified at present (diabetes, hypertension, obesity and smoking) do not give a good prediction of the risk of an individual developing CAD. Complex diseases such as CAD are caused by a combination of environmental and genetic factors, and altered molecular mechanisms. Network biology-based approaches have been previously used as powerful tools in studying and understanding the physical and functional interactions between these factors (1). Furthermore, these methods are being applied in the development of novel approaches for disease classification and the improved understanding of pathophenotypes (2).

Several studies suggesting that infections may predispose to CAD (3-8) are based on the evidence that infectious agents reside in the wall of atherosclerotic vessels (5), and seroepidemiological studies demonstrating an association between pathogen-specific immunoglobulin $\mathrm{G}$ antibodies and atherosclerosis. However, a comprehensive network analysis of infection and CAD has not been performed to understand the molecular associations between these two pathologies, specifically in humans.

A previous study by Gulbahce et al (9) provided several novel insights into viruses and diseases by constructing a viral disease network. Subsequently, numerous studies aiming to uncover the novel disease associations, in order to understand associations between clinical presentation and molecular networks, have been conducted (10-16). The present study aimed to use complex clinical phenotype information and molecular networks to elucidate the functional associations between infection, inflammation and CAD. Integration of discrete data sets from high throughput 
technologies with clinical phenotype information can potentially lead to the identification of the functional networks that respond to environmental and genetic factors.

Tools are often used with networks to graphically represent the nodes and edges, thus identifying the associations, interactions, co-expression, coregulation and modulations in normal and disease conditions. The addition of gene ontologies to these networks can provide a higher level of information of the alterations in biological processes/functions in diseases, thus may aid in the elucidation of causal associations between certain factors and disease. A similar study completed using macrophage-enriched metabolic networks in mice that were also conserved in humans identified potential causative mechanisms for several metabolic diseases (17). The current study identified that infections may trigger the networks of mechanisms including xenobiotic responses, cell surface anchoring and inflammation in myocardial infarction (MI). Furthermore, the analysis conducted additionally identified a simple and cost effective potential biomarker for identifying people at high risk of CAD and MI.

\section{Materials and methods}

The methodology adapted as presented in Fig. 1 was divided into four steps.

Step 1: Extraction of knowledge base. The human gene sets (flat files) were collected using the search terms, "infection", and "inflammation" from the UniProt database, which resulted in 475 and 814 genes (search conducted on Oct 31, 2013). For CAD, all 604 genes listed in the CAD Gene Database (http://www.bioguo.org/CADgene/) (18) were considered. Gene ontolgies $(\mathrm{GO})$ for all the genes were extracted from the UniProt flat files. In order to understand common molecular mechanisms and functions, GO terms of the three gene sets were matched. A unique list of GO terms was used for each gene set in each of the steps.

Step 2: Linking the experimental data to clinical phenotypes. In order to understand the role and molecular connections of infection, specifically of cytomegalovirus (CMV), 434 genes that were demonstrated to be differentially expressed upon infection of the human foreskin fibroblast cell line (CC-2509) with CMV (19) were investigated. Furthermore, 34 clinical terms associated with CAD extracted from the Unified Medical Language System (http://www.nlm.nih. gov/research/umls/) (Table I) were used as search terms for extracting corresponding genes from the UniProt database. Out of 34 clinical phenotypes, 23 resulted in 3,489 genes. The GO terms of all of these genes were combined and a unique list of 10,547 terms was generated. These terms associate CMV infection-mediated gene expression with clinical phenotypes.

Step 3: Identification of functional communicator ontologies (FCOs) and the highly networked clinical phenotype. In order to identify the FCOs which link different clinical phenotypes, 783 common GO terms from step 1 and step 2 were used. Due to the complexity of representing and evaluating a large network, parent ontologies termed as FCOs were used in the final construction of network using Cytoscape 3.0.2 software
Table I. The 34 medical terms associated with coronary artery disease extracted from the Unified Medical Language System.

Medical term

1. Tobacco abuse ${ }^{\mathrm{a}}$

2. Angina

3. Exercise stress test abnormal

4. Chest pain

5. Shortness of breath

6. Obesity

7. Coronary artery disease risk high

8. Hypertension

9. Coronary artery bypass graft ${ }^{\mathrm{a}}$

10. Organic heart disease

11. Heart

12. Angina stable

13. Epigastric pain ${ }^{\mathrm{a}}$

14. Myocardial infarction acute

15. Angina unstable

16. Orthopnea ${ }^{a}$

17. Coronary atherosclerotic heart disease

18. Cardiac arrest

19. Diabetic retinopathy

20. Lower extremity edema ${ }^{a}$

21. Congestive heart failure

22. Myocardial infarction

23. Cardiac catheterization ${ }^{\mathrm{a}}$

24. Electrocardiogram abnormal ${ }^{\mathrm{a}}$

25. Percutaneous transluminal coronary angioplasty planned $^{\mathrm{a}}$

26. Pyuria ${ }^{\mathrm{a}}$

27. Hypercholesterolemia

28. Diabetes mellitus

29. Atrial fibrillation new onset

30. Ischemic heart disease silent ${ }^{\mathrm{a}}$

31. Weight loss

32. Diabetes mellitus insulin dependent

33. Dyspnea paroxysmal nocturnal ${ }^{\mathrm{a}}$

34. Hyperlipidemia

${ }^{a}$ Did not elicit any hits in the UniProt database.

(http://www.cytoscape.org/). Network topology statistics such as node degree distribution and the average short path were used as parameters for selection of highly networked clinical phenotypes and FCOs.

Step 4: Expression analysis and validation in patients Identification of differentially expressed genes in patients. Based on the network parameters described in step 3, MI was identified to be a highly networked clinical phenotype. Therefore, the data set GSE48060 previously published by Suresh et al (20) was selected for further gene expression analysis. The raw microarray data was downloaded from the Gene Expression Ominibus (http://www.ncbi.nlm.nih.gov/geo/) and was analyzed using GeneSpring software, version 12.5 


\section{Methodology}

Step 1: Extract data from databases

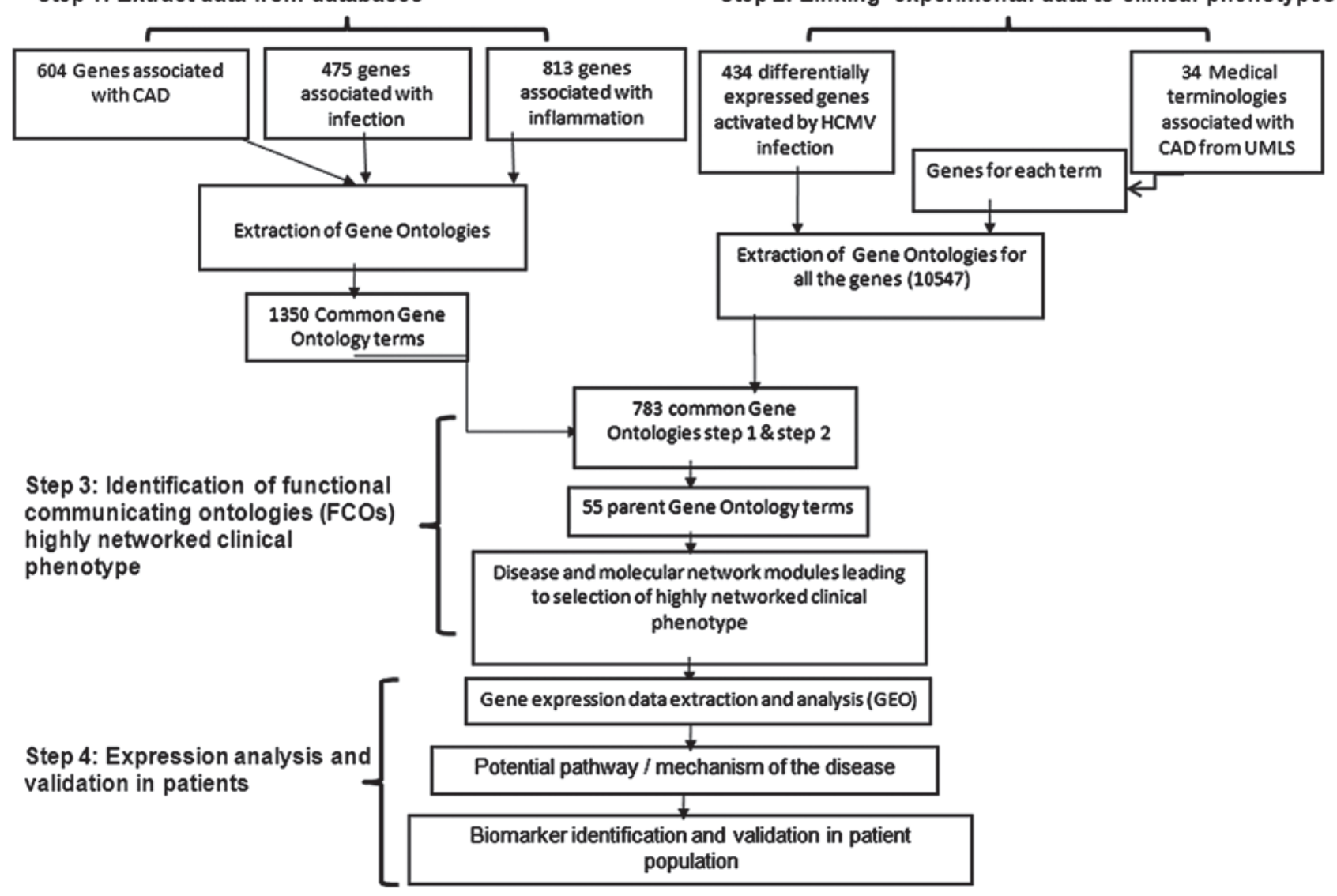

Figure 1. Methodology for identifying important pathways and associated biomarkers linking CAD, infection and inflammation. CAD, coronary artery disease; HCMV, human cytomegalovirus; UMLS, Unified Medical Language System.

(Agilent Technologies, Inc., Santa Clara, CA, USA). The GO terms of the differentially expressed genes were matched to $783 \mathrm{GO}$ terms in order to elucidate the potential mechanism of CMV infection leading to CAD. The gene(s) containing the matched GO terms were used for experimental validation in the selected patient population.

Selection of patient population for validation of potential biomarker. For validation of potential biomarker(s) patients enrolled in the Indian Atherosclerosis Research Study (IARS) were selected. The IARS is a prospective cohort study designed to investigate and understand the molecular basis of CAD in the Indian population. The design and details of data collection and the procedures used were described previously (21). The study was designed in accordance with the principles and the guidelines of World Medical Association Declaration of Helsinki and the Indian Council of Medical Research and approved by the Thrombosis Research Institute Ethics committee. Signed informed consent was obtained from all the participants who were enrolled from Narayana Institute of Cardiac Sciences (Bangalore, India) and the Asian Heart Institute and Research Centre (Mumbai, India). Between 2004 and 2006, 2,332 subjects were enrolled of which 772 (33\%) were patients with CAD as diagnosed by an angiogram. Out of these, 200 suffered from MI. These patients with MI were age and gender matched to 200 unaffected subjects (as indicated by a normal electrocardiogram) and the serum samples of all these subjects were used for biomarker assays. All blood samples were collected subsequent to overnight (12-14 h) fasting and the plasma and serum aliquots were stored at $-80^{\circ} \mathrm{C}$. Detailed information on the medical history, demographics and relevant information including a 3-generation pedigree was collected and recorded in a printed questionnaire subsequent to a personal interview. Diagnosis of diabetes and hypertension was based on medical reports while body mass index was calculated.

Telephone assessment of cardiac health status. Periodic telephone follow-up of all study participants was undertaken to obtain an update on the cardiac health status, with five rounds of follow-up completed. Follow-up was performed once every 18 months on average from the time of recruitment. Endpoints included non-fatal events (redo percutaneous transluminal coronary angioplasty/redo coronary artery bypass graft/heart attack/stroke) and fatal events (fatal heart attack/fatal stroke/sudden death/cardiac arrest). This information was verified through hospital records wherever available.

$\gamma$-glutamyl transferase-5 (GGT-5) assays and statistical analysis. The plasma GGT-5 concentration was measured using Flex Reagent Cartridges (Siemens Healthcare GmbH, Erlangen, Germany) in Dimension Xpand Plus Integrated Chemistry System (Siemens Healthcare GmbH). The inter assay coefficient of variation was $5.35 \%$. 
Table II. Number of genes, their ontologies and identified FCOs linking molecular data and clinical phenotypes.

\begin{tabular}{lccc}
\hline Category & $\begin{array}{c}\text { No. of } \\
\text { genes }\end{array}$ & $\begin{array}{c}\text { No. of gene } \\
\text { ontology terms }\end{array}$ & $\begin{array}{c}\text { No. of common } \\
\text { ontologies }\end{array}$ \\
\hline Coronary artery disease & 604 & 4277 & 783 \\
Infection & 475 & 2556 & 55 \\
Inflammation & 814 & 4525 & FCOs \\
Cytomegalovirus & 433 & 2263 & 8284 \\
UMLS (medical ontologies) & 3489 & & \\
\hline
\end{tabular}

FCOs, functional communicator ontologies; UMLS, unified Medical Language System.

Statistical analysis was conducted using SPSS, version 17.0 (SPSS, Inc., Chicago, IL, USA). Results were presented as the mean \pm standard error for the continuous variables. Student's independent t-test was used to test for mean differences in quantitative variables between the MI affected and unaffected subjects, while the $\chi^{2}$ test was used for testing the association of qualitative (discrete) variables with CAD. Hypertension, diabetes and smoking were considered as the conventional risk factors (CRFs). To assess the association of individual biomarkers and combinations, logistical regression analysis was performed. To assess the accuracy of discrimination by different models of GGT-5, receiver operating characteristics or C statistics analysis was performed. The statistical significance between the area under the curve (AUCs) was calculated using the DeLong method (22) using the R statistical program, version 3.2.1 (https://cran.r-project.org/). $\mathrm{P}<0.05$ was considered to indicate a statistically significant difference. For the same set of subjects, CMV experimental data (200 controls and 200 CAD-affected subjects) were used from a previous publication by our group (8).

\section{Results}

Functions as molecular communicators between different clinical phenotypes. As presented in Table II, 4,277 GO terms were identified for the CAD genes followed by 2,556 for infection and 4,525 for inflammation. These search terms resulted in 1,350 common GO terms in step 1 . In step 2, 2,263 unique GO terms were identified for the differentially expressed genes upon CMV infection and 8,284 for 23 clinical phenotypes. Furthermore 783 GO terms matched between step 1 and step 2 were identified, which represent potential links between diseases and molecular functions.

Network of clinical and molecular ontologies. In order to identify the molecular functions networking with 23 clinical phenotypes, 55 parent ontologies of 783 GO terms were extracted, which were termed FCOs. As presented in Fig. 2, different FCOs and clinical phenotypes are associated with various degrees of connectivity. Using node degree (ND) and average short path (ASP) analysis (Table III), it was identified that FCOs such as apoptosis (ND:14 and ASP:1.8) and cell adhesion (ND:12, ASP:1.9) were two important molecular functions. These functions were highly networked with different clinical phenotypes, followed by mitochondria, regulation of apoptosis, cytokine activity, lipid metabolism, viral entry and immune response. These functions are in concordance with viral infections. Of the 23 clinical phenotypes, MI (ND:15, ASP:2.5) and cardiac arrest (ND:10, ASP:2.3) were observed to be highly networked with several FCOs (Table IV). MI was considered for further analysis as it was highly connected with important FCOs including viral infections, cell adhesion, entry of virus into host cell, apoptosis, regulation of apoptosis, stress response and immune response (Table IV).

The network of clinical terms and FCOs also suggest that there are common mechanisms and sub-phenotype specific molecular signals which may serve an important role in disease onset and progression. As presented in Table IV, the carbohydrate metabolism appears to be a specific molecular function associated with cardiac arrest and lysosome function for congestive heart failure.

Fusion of global gene expression and FCOs for mechanism and biomarker discovery. From the diseasome-function network (Fig. 2) for further understanding of the potential mechanism of infection in CAD, the gene expression data and FCOs were brought together. The global gene expression data analysis of the MI dataset GSE48060 (19) indicated 243 differentially expressed probe sets, of which 170 genes contained GO, gene symbol and Entrez gene ID information. The 63 GO terms of these differentially expressed genes were matched with $783 \mathrm{GO}$ terms from step 3. As presented in the Fig. 3A, the four matching GO terms were: Anchored to membrane, external side of plasma membrane, xenobiotic metabolic process and regulation of inflammatory process. These molecular functions and processes suggest that persistent infection(s) by CMV may lead to increased inflammation and activation of xenobiotic mechanisms (Fig. 3B). Genes associated with these four GO terms and were further analyzed, and it was identified that the $\gamma$-glutamyl transferase family of genes (GGT5, GGTL3 and GGT4) were involved in all 4 of the functions, and additional genes such as TNFAIP3 interacting protein 1 and complement receptor 1 were associated with a minumum of 2. Therefore, for further analysis GGT5 was considered as a potential biomarker for assessment in patient subjects.

\section{Biomarker validation}

Patient baseline characteristics. As presented in Table V, mean differences of body mass index, waist:hip ratio, waist circumference and triglycerides were not identified to be 
Table III. Highly connected functional communicator ontologies based on node degree distribution and average short path in the network.

Gene ontology term

Average short path

Node degree

Apoptosis

Cell adhesion

Mitochondrion

Regulation of apoptosis

Cytokine activity, endocytosis

Lipid metabolism, stress-activated MAPK cascade, endosome, carbohydrate metabolism, immune response

Lysosome

Viral life cycle, stress response, MAPKKK cascade

Entry of virus into host cell

Response to external stimulus, B cell activation, induction of apoptosis via death domain receptors, entry into host cell, phosphoinositide 3-kinase cascade Regulation of c-Jun N-terminal kinase cascade

Complement activation-classical pathway, hormone secretion, phagocytosis, cytokine production, T cell activation, cytokine secretion, Janus kinase-signal transducer and activator of transcription cascade

Complement activation, cytokine biosynthesis, humoral defense mechanism \& immune response, protein metabolism, regulation of $\mathrm{I}-\kappa \mathrm{B}$ kinase $/ \mathrm{NF}-\kappa \mathrm{B}$ cascade, regulation of MAPKKK cascade, response to biotic stimulus

$\begin{array}{cr}1.86440678 & 14 \\ 1.93220339 & 12 \\ 1.96610169 & 11 \\ 2 & 10 \\ 2.06779661 & 8 \\ 2.10169492 & 7\end{array}$

2.13559322

6

$2.16949153 \quad 5$

2.20338983

2.23728814

2.20338983

2.27118644

2.30508475

MAPK, mitogen-activated protein kinase; MAPKKK, MAPK kinase kinase.

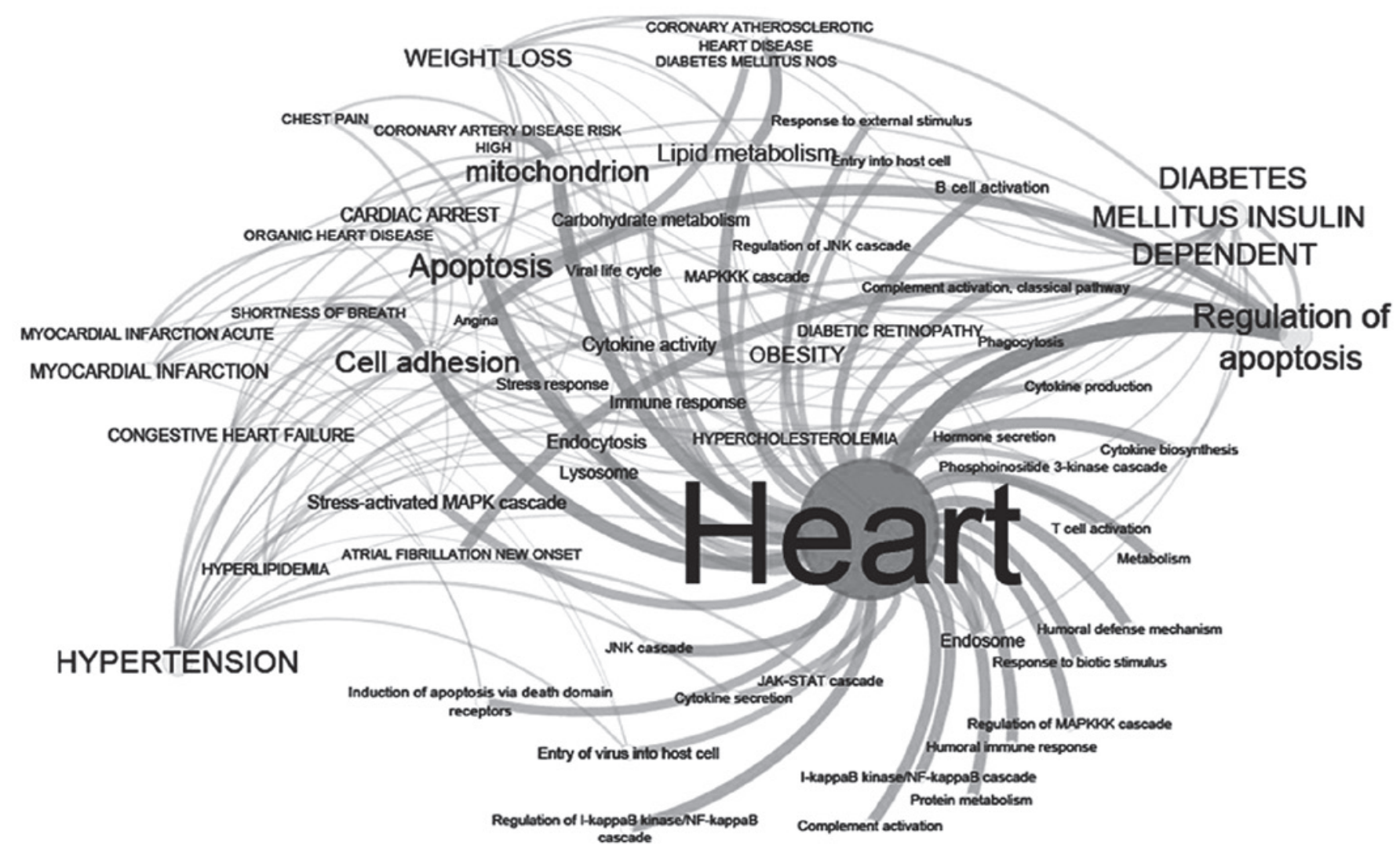

Figure 2. Network of 55 FCOs and 23 clinical phenotypes. The nodes indicate the FCOs and clinical phenotypes for coronary artery disease, and the edges indicate the associations between FCOs and clinical phenotypes. FCOs, functional communicator ontologies; JNK, c-Jun N-terminal kinase; MAPK, mitogen-activated protein kinase; MAPKKK, MAPK kinase kinase; JAK, Janus kinase; STAT, signal transducer and activation of transcription; NF, nuclear factor. 
Table IV. Top 5 highly networked clinical terminologies based on node degree distribution.

\begin{tabular}{|c|c|c|}
\hline Name & Average short path & Node degree (functional communicator ontologies) \\
\hline $\begin{array}{l}\text { MI including } \\
\text { acute MI }\end{array}$ & 2.52542373 & $\begin{array}{l}14 \text { (entry of virus into host cell, endosome, apoptosis, regulation of apoptosis, } \\
\text { stress-activated MAPK cascade, stress response, immune response, immunity, } \\
\text { cell adhesion, endocytosis, apoptosis via death domain receptors, inflammation, } \\
\text { regulation of inflammation, external side of membrane) }\end{array}$ \\
\hline Cardiac arrest & 2.37288136 & $\begin{array}{l}10 \text { (viral life cycle, cell adhesion, stress response, apoptosis, regulation of } \\
\text { apoptosis, cytokine activity, immune response, mitochondrion, carbohydrate } \\
\text { metabolism, stress-activated MAPK cascade) }\end{array}$ \\
\hline $\begin{array}{l}\text { Congestive heart } \\
\text { failure }\end{array}$ & 2.47457627 & $\begin{array}{l}7 \text { (cell adhesion, apoptosis, regulation of apoptosis, apoptosis by death domain } \\
\text { receptors, mitochondrion, stress-activated MAPK cascade, lysosome) }\end{array}$ \\
\hline Chest pain & 2.6779661 & 3 (cell adhesion, apoptosis, mitochondrion) \\
\hline Angina & 2.98305085 & 1 (regulation of apoptosis) \\
\hline
\end{tabular}

MI, myocardial infarction; MAPK, mitogen-activated protein kinase.

\section{A}

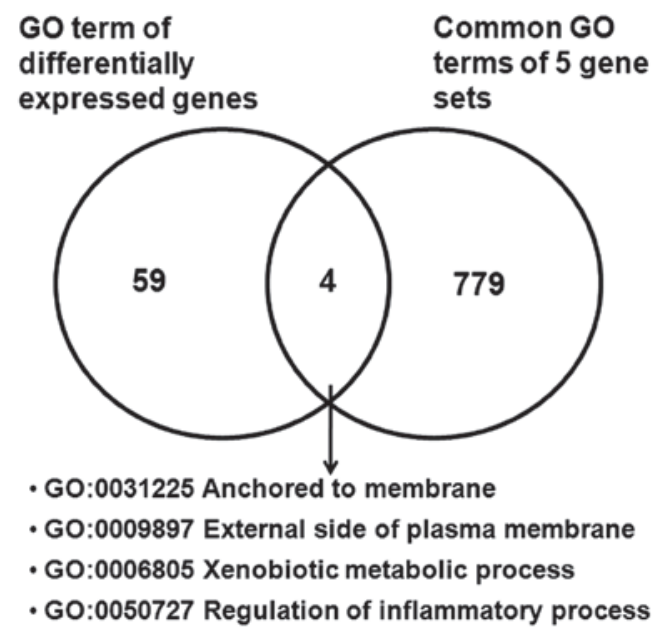

B

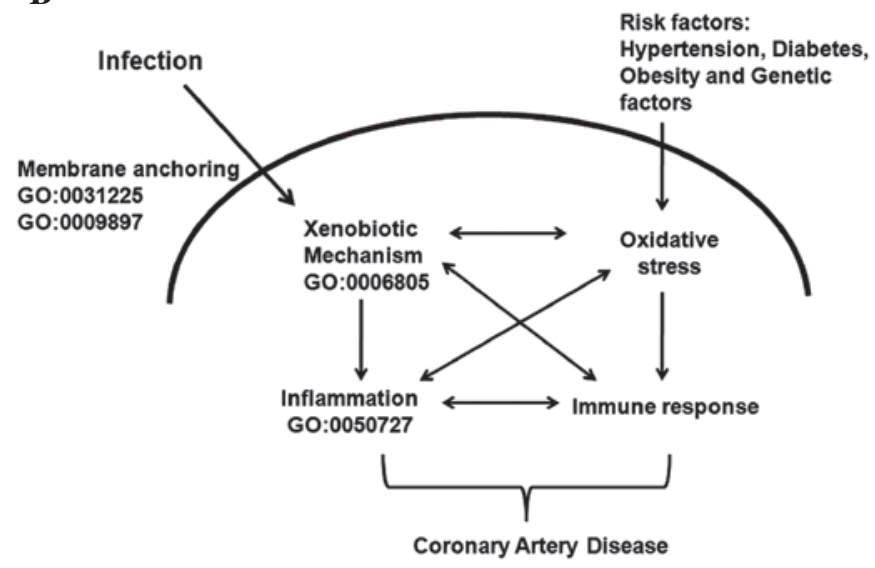

Figure 3. (A) Common GO terms from global gene expression analysis and 78 GO terms. (B) Potential molecular mechanism of the association between infection, inflammation and coronary artery disease. GO, gene ontology.

significantly different between MI-affected and -unaffected subjects, whereas the mean of high density lipoprotein (HDL) was observed to be significantly higher in unaffected subjects $(\mathrm{P}=0.011)$. The frequency of diabetes, hypertension, smoking and usage of prescription medication including statins, beta blockers, fibrates, calcium channel blockers, angiotensin-converting enzyme inhibitors, hypoglycemic agents, nitrates and anti-platelet agents was significantly increased in MI-affected subjects. In addition, total cholesterol and low density lipoprotein (LDL) were observed to be at low levels in affected subjects, which may be due to the greater usage of statins in this group. Additional biomarkers including GGT-5, CMV and CMV-NA titers were also observed to be significantly increased in patients with $\mathrm{MI}$ in comparison with unaffected individuals.

Association of GGT-5 in patients with MI. In the logistical regression analysis (Table VI) for the biomarkers (GGT-5, CMV and CMV-NA titers), it was identified that GGT-5 alone was associated with an odds ratio of 1.947 (95\% CI: 1.14-3.31, $\mathrm{P}=0.014)$, which was greater than for the other markers. The odds ratios for the combination of GGT-5 + CMV-NA titers was 1.872 (95\% CI, 1.03-3.41; P=0.041), and GGT-5 + CMV was 2.226 (95\% CI, 1.26-3.92; $\mathrm{P}=0.006)$. When all three biomarkers were added into the model the odds ratio was 2.133 (95\% CI, 1.12-4.03; $\mathrm{P}=0.020$ ) which further increased to 2.338 (95\% CI, 1.17-4.64; $\mathrm{P}=0.015$ ) upon addition of $\mathrm{CRFs}$ (waist circumference, hypertension, diabetes and smoking) and lipids (HDL and LDL). In the final model, as GGT-5 is known to be associated with alcohol consumption, this was adjusted for and it was identified that the odds ratio increased to 2.561 (95\% CI, 1.27-5.15; $\mathrm{P}=0.009$ ). The $\mathrm{C}$-statistics analysis (AUC) indicated that CRFs alone had a AUC of 0.53 (95\% CI, 0.47-0.58) that increased in the final model to 0.711 (95\% CI, 0.64-0.77). This increase was significant as measured using the DeLong method with $\mathrm{P}=0.005$.

\section{Discussion}

In order to prevent, diagnose and treat disease, there is a requirement to integrate data from different sources (biological experiments and clinopathological information), which will be stored in different formats depending on the 
Table V. Base line characteristics of patient group selected for study.

\begin{tabular}{|c|c|c|c|}
\hline Variables & Unaffected $(n=200)$ & Affected (MI, n=200) & P-value \\
\hline $\operatorname{Age}^{\mathrm{a}}$ & $51.88 \pm 0.74$ & $51.90 \pm 0.74$ & 0.98 \\
\hline Gender (Male) & $157(78.5 \%)$ & $157(78.5 \%)$ & 0.548 \\
\hline Gender (Female) & $43(21.5 \%)$ & $43(21.5 \%)$ & \\
\hline Body mass index $\mathrm{kg} / \mathrm{m}^{2}$ & $25.21 \pm 0.284$ & $25.69 \pm 0.280$ & 0.22 \\
\hline Waist:hip ratio ${ }^{a}$ & $0.93 \pm 0.005$ & $0.94 \pm 0.005$ & 0.28 \\
\hline Waist circumference ${ }^{\mathrm{a}}(\mathrm{cm})$ & $90.61 \pm 0.73$ & $90.35 \pm 0.85$ & 0.81 \\
\hline Total cholesterol ${ }^{\mathrm{a}}$ (mg/dl) & $177.41 \pm 2.83$ & $150.49 \pm 2.64$ & $1.54^{\mathrm{a}} 10^{-11}$ \\
\hline Triglycerides $^{\mathrm{a}}(\mathrm{mg} / \mathrm{dl})$ & $160.71 \pm 7.716$ & $159.44 \pm 5.10$ & 0.89 \\
\hline High-density lipoprotein ${ }^{\mathrm{a}}$ (mg/dl) & $37.58 \pm 0.66$ & $35.29 \pm 0.60$ & 0.011 \\
\hline Low-density lipoprotein ${ }^{\mathrm{a}}$ (mg/dl) & $110.47 \pm 2.53$ & $83.30 \pm 2.35$ & $3.85^{\mathrm{a}} 10^{-14}$ \\
\hline Smoking, n (\%) & $58(29.0 \%)$ & $94(47.0 \%)$ & $1.49^{\mathrm{a}} 10^{-4}$ \\
\hline Alcohol consumption, n (\%) & $42(21.0 \%)$ & $27(13.5 \%)$ & 0.032 \\
\hline Hypertension, n (\%) & $50(25.0 \%)$ & $82(41.0 \%)$ & $4.72^{\mathrm{a}} 10^{-4}$ \\
\hline Diabetes mellitus, n (\%) & $48(24.0 \%)$ & $88(44.0 \%)$ & $1.76^{\mathrm{a}} 10^{-5}$ \\
\hline Statin, n (\%) & $8(4.0 \%)$ & $137(68.5 \%)$ & $1.88^{\mathrm{a}} 10^{-46}$ \\
\hline Beta blocker, n (\%) & $26(13.0 \%)$ & $124(62.0 \%)$ & $2.68^{\mathrm{a}} 10^{-25}$ \\
\hline Fibrate, n (\%) & $1(0.5 \%)$ & $8(4.0 \%)$ & 0.018 \\
\hline Calcium channel blocker, n (\%) & $20(10.0 \%)$ & $51(25.5 \%)$ & $1.40^{\mathrm{a}} 10^{-17}$ \\
\hline ACE inhibitor, $\mathrm{n}(\%)$ & $15(7.5 \%)$ & $87(43.5 \%)$ & $1.40^{\mathrm{a}} 10^{-17}$ \\
\hline Hypoglycemic agents, n (\%) & $38(19.0 \%)$ & $63(31.5 \%)$ & 0.003 \\
\hline Nitrate, n (\%) & $1(0.5 \%)$ & $86(43.0 \%)$ & $7.09^{\mathrm{a}} 10^{-30}$ \\
\hline Antiplatelet, n (\%) & $7(3.5 \%)$ & $177(88.5 \%)$ & $1.24^{\mathrm{a}} 10^{-76}$ \\
\hline \multicolumn{4}{|l|}{ Biomarkers } \\
\hline $\mathrm{GGT}^{\mathrm{a}} \mathrm{U} / 1$ & $32.05 \pm 0.97$ & $36.05 \pm 1.25$ & 0.011 \\
\hline $\mathrm{CMV}^{\mathrm{a}}$ & $9.69 \pm 0.514$ & $12.55 \pm 0.66$ & 0.001 \\
\hline CMV-NA titres ${ }^{\mathrm{b}}$ & $4.67 \pm 0.052$ & $4.92 \pm 0.074$ & 0.008 \\
\hline
\end{tabular}

${ }^{\text {aMean }} \pm$ standard error; ${ }^{b}$ Log-transformed mean \pm standard error (retransformed mean). Significance was determined by Student's t-test for variables expressed as the mean \pm standard error and by the $\chi^{2}$ test for variables expressed as percentages. CMV data previously presented (8). MI, myocardial infarction; ACE, angiotensin-converting enzyme; GGT, $\gamma$-glutamyl transpeptidase; CMV, cytomegalovirus; CMV-NA, CMV-neutralizing antibody.

technologies used. This integration of data will provide an improved understanding of disease-disease associations, gene prioritization, function prediction and significant comorbidity effects in associated diseases (10-17). The current study developed, to the best of our knowledge for the first time, a novel translational informatics approach in combining molecular and clinical information for translational research. One of the key aspects of CAD research has been the identification of potential biomarkers for risk prediction, with infection having been identified as an important factor (1-7,23-29). However, the molecular and functional associations between the infection (by CMV) and CAD remain to be fully understood. The current study used data stored in molecular and clinical databases. The 55 FCOs (Table II) associated with 23 clinical phenotypes indicates the system-level molecular associations between diseases and molecular functions (Fig. 2 and Table III). This network may provide a platform for exploring the associations between phenotype and disease from a genetic to a functional level. The network statistics analysis suggested that important FCOs such as apoptosis, cell adhesion and lipid metabolism serve important roles in CAD (Table III). These functions may represent the associations between pathogen-induced injury and CAD onset or progression. Cell adhesion of virus and entry into endothelial cells may be the first steps towards vessel wall injury (30). Furthermore, it has been suggested that the inflammatory response to injury, dysregulation of lipid metabolism and the presence of activated macrophages and T-cells may lead to the initiation of atherosclerotic lesions. At the diseasome level, the highly networked disease MI was observed to network with important FCOs including entry of virus into host cell, apoptosis, stress response and immune response, which are also associated with infections (Table IV). A previous study suggested that infections may serve an important role in MI (31), which may potentially be due to modulation of the above pathways (Fig. 3 and Table V). This approach identified molecular functions associated with clinical phenotypes, and those associated with unique functions including carbohydrate metabolism specifically associated with cardiac arrest (Table IV). Furthermore, by combining 783 common 
Table VI. Logistical regression analysis for the association between GGT-5 and myocardial infarction in combination with CMV infection markers.

\begin{tabular}{lrr}
\hline Model & Odds ratio (95\% CI) & AUC (95\% CI) \\
\hline CRFs & - & $0.530(0.47-0.58)^{\mathrm{d}}$ \\
CMV & $1.31(1.04-1.63)^{\mathrm{c}}$ & $0.593(0.53-0.65)^{\mathrm{b}}$ \\
CMV-NA titres & $1.457(1.10-1.92)^{\mathrm{b}}$ & $0.587(0.52-0.65)^{\mathrm{b}}$ \\
GGT & $1.947(1.14-3.310)^{\mathrm{c}}$ & $0.570(0.51-0.62)^{\mathrm{c}}$ \\
GGT + CMV-NA titres & $1.872(1.03-3.41)^{\mathrm{c}}$ & $0.604(0.54-0.67)^{\mathrm{a}}$ \\
GGT + CMV & $2.226(1.26-3.92)^{\mathrm{b}}$ & $0.620(0.56-0.67)^{\mathrm{a}}$ \\
GGT + CMV-NA titres + CMV & $2.133(1.12-4.03)^{\mathrm{c}}$ & $0.633(0.57-0.69)^{\mathrm{a}}$ \\
GGT + CMV-NA titres + CMV + CRFs + lipids & $2.338(1.17-4.64)^{\mathrm{c}}$ & $0.704(0.64-0.76)^{\mathrm{a}}$ \\
GGT + CMV-NA titres + CMV + CRFs + lipids + alcohol consumption & $2.561(1.27-5.15)^{\mathrm{b}}$ & $0.711(0.64-0.77)^{\mathrm{a}}$ \\
\hline
\end{tabular}

${ }^{\mathrm{a}} \mathrm{P}<0.001,{ }^{\mathrm{b}} \mathrm{P}<0.01,{ }^{\mathrm{c}} \mathrm{P}<0.05,{ }^{\mathrm{d}} \mathrm{P}>0.05$. CRFs include waist circumference, hypertension, diabetes and smoking. The Hosmer lemshow test was performed for all the models and those with $\mathrm{P}<0.05$ are reported here. CMV data previously presented (8). GGT, $\gamma$-glutamyl transpeptidase; $\mathrm{CMV}$, cytomegalovirus; CI, confidence interval; AUC, area under the curve; CRFs, conventional risk factors; CMV-NA, CMV-neutralizing antibody.

GO terms between different mechanisms with the differentially expressed gene profiles of patients with MI, 4 important molecular functions were indicated, leading to the identification of GGT-5 as a potential biomarker (Tables V and VI). GGT-5 has been previously demonstrated to be an important marker for the onset of metabolic syndrome and predicts incidence of cardiovascular disease (32). In the current study, GGT-5 was demonstrated to be involved in the four identified GO terms including xenobiotic response, and it is suggested that in addition to its previously established role in oxidative stress and metabolic syndrome (33), GGT-5 may serve an important role in CAD predisposition (Fig. 3B).

Previous studies have demonstrated the association of infections and CAD, and additionally localization of infectious agents in the plaque area (1-7,23-29), suggesting that these infections may contribute to increases in inflammation in the plaque area. It has been previously suggested that indirect upregulation of inflammatory molecules may serve a role in CMV infection (3). However, in the current study (Fig. 3A and B), it was observed that active infection by CMV may trigger viral entry pathways including cell adhesion/plasma membrane adhesion stimulating xenobiotic the mechanism, an increase apoptosis, inflammation and modulation of immunity. This may explain failure of antibiotic trials for CAD $(33,34)$, as infection may lead to modulation of the xenobiotic mechanism (Fig. 3B), resulting in ineffectiveness of the drugs. Infection has also been demonstrated to accelerate atherosclerosis in hyperlipidemic animal models (25-29), suggesting that infections in addition to risk factors including obesity, diabetes and hypertension may increase the oxidative stress which is known to increase inflammation. Therefore, infection is able to stimulate a cascade of molecular events (Fig. 3B) leading to imbalanced inflammatory and immune responses. Persistent infections which trigger long term alterations in the abovementioned molecular mechanisms are able to potentiate disease progression and development from lesion generation to plaque formation, which may explain the presence of infectious agents in plaques. The methods used in the current study for fusion of clinical and molecular data may aid in the advancement of patient care and further research.

In conclusion, an integrative translational informatics approach was established in the current study, which may aid in the understanding of the molecular mechanisms in CAD, and additionally identify potential biomarkers for use in risk stratification. However, it is necessary to perform biomarker assays in a larger patient population to validate the observations of the current study.

\section{Acknowledgements}

The current study was supported by the Department of Biotechnology, Ministry of Science and Technology, Government of India (grant no. BT/01/CDE/08/07), the Tata Social Welfare Trust, India (grant no. TSWT/IG/SNB/JP/Sdm) and the Bharati Foundation, India (grant no. 005/2012-2013). Sponsors had no role in the design, conduct, sample collection, analysis and interpretation of the data or in the preparation, review or approval of the manuscript. The authors would like to thank all investigators, staff and administrative teams and participants of IARS at Narayana Institute of Cardiac Sciences, Bangalore (India) and the Asian Heart Institute, Mumbai (India) for their contributions. The authors would also like to thank the patients and their family members for participating in the study.

\section{References}

1. Cho DY, Kim YA and Przytycka TM: Chapter 5: Network biology approach to complex diseases. PLOS Comput Biol 8: e1002820, 2012.

2. Loscallzo J, Kohanne I and Barabasi AL: Human disease classification in the post genomic era: A complex systems approach to human pathobiology. Mol Sys Biol 3: 124, 2007.

3. Epstein SE, Zhu J, Najafi AH and Burnett MS: Insights into the role of infection in atherogenesis and in plaque rupture. Circulation 119: 3133-3141, 2009.

4. Epstein SE, Zhou YF and Zhu J: Infection and atherosclerosis: Emerging mechanistic paradigms. Circulation 100: e20-e28, 1999. 
5. Rosenfield ME and Campbell LA: Pathogenesis and atherosclerosis: Update on potential contribution of multiple infectious organisms to pathogenesis of atherosclerosis. Thromb Haemost 106: 858-867, 2011

6. Shah PK: Link between infection and atherosclerosis: Who are the culprits: Virises, bacteria, both, or neither? Circulation 103 5-6, 2001.

7. Stassen FR, Vainas $\mathrm{T}$ and Bruggeman CA: Infection and atherosclerosis. An alternative view on an outdated hypothesis. Pharmacol Rep 60: 85-92, 2008.

8. Mundkur LA, Rao VS, Hebbagudi S, Shanker J, Shivanandan H, Nagaraj RK and Kakkar VV: Pathogen burden, cytomegalovirus infection and inflammatory markers in the risk of premature coronary artery disease in individuals of Indian origin. Exp Clin Cardiol 17: 63-68, 2012.

9. Gulbahce N, Yan H, Dricot A, Padi M, Byrdsong D, Franchi R, Lee DS, Rozenblatt-Rosen O, Mar JC, Calderwood MA, et al: Viral perturbations of host networks reflect disease etiology. PLoS Comput Biol 8: e1002531, 2012.

10. Lee DS, Park J, Kay KA, Christakis NA, Oltvai ZN and Barabási AL: The implications of human metabolic network topology for disease comorbidity. Proc Natl Acad Sci USA 105 9880-9885, 2008.

11. Goh KI, Cusick ME, Valle D, Childs B, Vidal M and Barabási AL: The human disease network. Proc Natl Acad Sci USA 104 8685-8690, 2007.

12. Linghu B, Snitkin ES, Hu Z, Xia Y and Delisi C: Genome-wide proirotization of disease genes and identification of disease-disease associations from an integrated human functional linkage network. Genome Biol 10: R91, 2009.

13. Janić $\mathrm{C}$ and Pržulj N: Biological function through network topology: A survey of the human diseasome. Brief Funct Genomics 11: 522-532, 2012.

14. Emmert-Streib F, Tripathi S, de Matos Simoes R, Hawwa AF and Dehmer M: The human disease network. Opportunities for classification, diagnosis and prediction of disorders and disease genes. Systems Biomedicine 1: 20-28, 2013.

15. Piro RM and Di Cunto F: Computational approached to disease-gene prediction: Rationale, classification and successes. FEBS J 279: 678-696, 2012.

16. Yu S, Falck T, Daemen A, Tranchevent LC, Suykens JA, De Moor B and Moreau Y: $\mathrm{L}_{2}$-norm multiple kernel learning and its application to biomedical data fusion. BMC Bioinformatics 11: 309, 2010.

17. Chen Y, Zhu J, Lum PY, Yang X, Pinto S, MacNeil DJ, Zhang C, Lamb J, Edwards S, Sieberts SK, et al: Variations in DNA molecular networks that cause disease. Nature 452: 429-435, 2008

18. Liu H, Liu W, Liao Y, Cheng L, Liu Q, Ren X, Shi L, Tu X, Wang QK and Guo AY: CADgene: A comprehensive database for coronary artery disease genes. Nucleic Acids Res 39 (Database issue): D991-D996, 2011.

19. Wang A, Ren L and Li H: A systemic network triggered by human cytomegalovirus entry. Adv Virol 2011: 262080, 2011.
20. Suresh R, Li X, Chiriac A, Goel K, Terzic A, Perez-Terzic C and Nelson TJ: Transcriptome from circulating cells suggests dysregulated pathways associated with long-term recurrent events following first-time myocardial infarction. J Mol Cell Cardiol 74: 13-21, 2014.

21. Shanker J, Mitra A, Rao VS, Mundkur L, Dhanalakshmi B, Hebbagodi S and Kakkar VV: Rationale, design \& preliminary findings of the Indian atherosclerosis research study. Indian Heart J 62: 286-295, 2010.

22. DeLong ER, DeLong DM and Clarke-Perason DL: Comparing the areas under two or more correlated receiver operating characteristics curves: A nonparametric approach. Biometrics 44: 837-845, 1988.

23. Ji YN, An L, Zhan P and Chen XH: Cytomegalovirus infection and coronary heart disease risk: A meta-analysis. Mol Biol Rep 39: 6537-6546, 2012.

24. Span AH, Van Boven CP and Bruggeman CA: The effect of cytomegalovirus infection on the adherence of polymorphonuclear leucocytes to endothelial cells. Eur J Clin Invest 19: 542-548, 1989.

25. Naghavi M, Wyde P, Litovsky S, Madjid M, Akhtar A, Naguib S, Siadaty MS, Sanati S and Casscells W: Influenza infection exerts prominent inflammatory and thrombotic effects on the atherosclerotic plaques of apolipoprotein E-deficient mice. Circulation 107: 762-768, 2003.

26. Hsich E, Zhou YF, Paigen B, Johnson TM, Burnett MS and Epstein SE: Cytomegalovirus infection increases development of atherosclerosis in apolipoprotein-E knockout mice. Atherosclerosis 156: 23-28, 2001.

27. Burnett MS, Gaydos CA, Madico GE, Glad SM, Paigen B, Quinn TC and Epstein SE: Atherosclerosis in apoE knockout mice infected with multiple pathogens. J Infect Dis 183: 226-231, 2001.

28. Ezzahiri R, Neilssen-Vrancken HJ, Kurvers HA, Stassen FR, Vliegen I, Grauls GE, van Pul MM,Kitslaar PJ and Bruggeman CA: Chlamydophila pneumonia (Chlamidia pneumoniae) accelerates the formation of complex atherosclerotic lesions in apo E3-Leiden mice. Cardiovasc Res 56: 269-276, 2002.

29. Ezzahiri R, Stassen FR, Kurvers HA, van Pul MM, Kitslaar PJ and Bruggemann CA: Chlamydia pneumoniae infection induces an uns- atherosclerotic plaque phenotype in LDL-receptor, ApoE double knockout mice. Eur J Vasc Endovasc Surg 26: 88-95, 2003.

30. Janeway CA Jr, Travers P, Walport M and Shlomchik MJ. The front line of host defense. In: Immunobiology: The Immune System in Health and Disease. 5th edition. Garland Science, New York, pp295-340, 2001.

31. Smeeth L, Thomas SL, Hall AJ, Hubbard R, Farrington P and Vallance P: Risk of myocardial infarction and stroke after acute infection or vaccination. N Engl J Med 351: 2611-2618, 2004.

32. Anderson JL and Muhlestein JB: Antiobiotic trials for coronary heart disease. Tex Heart Inst J 31: 33-38, 2004.

33. Dhingra R, Gona P, Wang TJ, Fox CS, D'Agostino RB Sr and Vasan RS: Serum gamma glutamyl transferase and risk of heart failure in the community. Atheroscler Thromb Vasc Biol 30: $1855-1860,2010$

34. Gryaston JT: Antiobiotic treatment of atherosclerotic cardiovascular disease. Circulation 107: 1228-1230, 2003. 